\title{
A prospective study of the importance of enteric fever as a cause of non-malarial febrile illness in patients admitted to Chittagong Medical College Hospital, Bangladesh
}

Rapeephan R. Maude1, Aniruddha Ghose ${ }^{2}$, Rasheda Samad², Hanna K. de Jong ${ }^{3}$, Masako Fukushima ${ }^{4}$, Lalith Wijedoru', Mahtab Uddin Hassan², Md Amir Hossain², Md Rezaul Karim², Abdullah Abu Sayeed², Stannie van den Ende ${ }^{3}$, Sujat Pal' ${ }^{2}$ A. S. M. Zahed ${ }^{2}$, Wahid Rahman², Rifat Karnain², Rezina Islam², Dung Thi Ngoc Tran ${ }^{5}$, Tuyen Thanh Ha ${ }^{5}$, Anh Hong Pham ${ }^{5}$, James I. Campbell, ${ }^{5,6}$, H. Rogier van Doorn ${ }^{5,6}$, Richard J. Maude ${ }^{1,6}$, Tom van der Poll ${ }^{3}$, W. Joost Wiersinga ${ }^{3}$, Nicholas P. J. Day ${ }^{1,6}$, Stephen Baker ${ }^{5,6,7}$, Arjen M. Dondorp ${ }^{1,6}$, Christopher M. Parry ${ }^{1,4,7,8^{*}}$ and Md Abul Faiz ${ }^{1,9}$

\begin{abstract}
Background: Fever is a common cause of hospital admission in Bangladesh but causative agents, other than malaria, are not routinely investigated. Enteric fever is thought to be common.

Methods: Adults and children admitted to Chittagong Medical College Hospital with a temperature of $\geq 38.0{ }^{\circ} \mathrm{C}$ were investigated using a blood smear for malaria, a blood culture, real-time PCR to detect Salmonella Typhi, S. Paratyphi A and other pathogens in blood and CSF and an NS1 antigen dengue ELISA.

Results: We enrolled 300 febrile patients with a negative malaria smear between January and June 2012: 156 children (aged $\leq 15$ years) and 144 adults with a median (interquartile range) age of 13 (5-31) years and median (IQR) illness duration before admission of five (2-8) days. Clinical enteric fever was diagnosed in 52 patients (17.3\%), lower respiratory tract infection in 48 (16.0\%), non-specific febrile illness in 48 (16.0\%), a CNS infection in 37 patients (12.3\%), urinary sepsis in 23 patients (7.7\%), an upper respiratory tract infection in 21 patients (7.0\%), and diarrhea or dysentery in 21 patients (7.0\%). Malaria was still suspected in seven patients despite a negative microscopy test. S. Typhi was detected in blood by culture or PCR in $34(11.3 \%)$ of patients. Of note Rickettsia typhi and Orientia tsutsugamushi were detected by PCR in two and one patient respectively. Twenty-nine (9\%) patients died during their hospital admission (15/160 (9.4\%) of children and 14/144 (9.7\%) adults). Two of 52 (3.8 \%) patients with enteric fever, $5 / 48$ (10.4\%) patients with lower respiratory tract infections, and 12/37 (32.4\%) patients with CNS infection died.

\footnotetext{
* Correspondence: chris.parry@lshtm.ac.uk

This work was presented in part at the 24th European Congress in Clinical

Microbiology and Infectious Diseases May 2014, Barcelona, Spain.

'Mahidol-Oxford Tropical Medicine Research Unit (MORU), Faculty of Tropical

Medicine, Mahidol University, Bangkok, Thailand

${ }^{4}$ Clinical Sciences, Liverpool School of Tropical Medicine, Liverpool, UK

Full list of author information is available at the end of the article
} 
(Continued from previous page)

Conclusion: Enteric fever was confirmed in $11.3 \%$ of patients admitted to this hospital in Bangladesh with nonmalaria fever. Lower respiratory tract and CNS infections were also common. CNS infections in this location merit more detailed study due to the high mortality.

Keywords: Non-malaria febrile illness, Bangladesh, Enteric fever

\section{Background}

Febrile illnesses are a common cause of admission to hospitals in resource limited settings but the limited availability of routine diagnostic microbiology testing means that the causes are often incompletely understood. Intensive diagnostic studies performed over short periods have proved to be a useful approach to determine the relative importance and resistance patterns of common pathogens and the identification of new infections [1-4]. A greater understanding the clinical epidemiology of local infections enables public health authorities to focus limited resources.

In Chittagong Division, Bangladesh, malaria is an important reason for people with fever to seek medical advice and hospital admission and is generally diagnosed using a malaria blood smear and/or a rapid diagnostic test [5]. For patients in whom malaria has been excluded, enteric (typhoid) fever is believed to be common and dominated by antimicrobial resistant organisms [6-13]. The importance of other diseases, such as melioidosis, rickettsiosis and leptospirosis, are not well defined [14]. There is serological evidence of exposure to Burkholderia pseudomallei (causative agent of melioidosis), Orientia tsutsugumushi and Rickettisa typhi (the causative agent of rickettsiosis) but very few reports of confirmed illness $[15,16]$.

We conducted a prospective study to assess the etiology of non-malarial febrile illness in adults and children admitted to Chittagong Medical College Hospital, a 1000bed regional teaching hospital. Our aim was to determine the proportion of patients with enteric fever. We also sought to determine the range of other clinical syndromes, causative agents and to document patient outcomes. An evaluation of typhoid rapid diagnostic tests in the patients in this study has been reported previously [17].

\section{Methods}

\section{Study site and population}

Chittagong Medical College Hospital $(\mathrm{CMCH})$ is a 1000-bed teaching hospital in Chittagong Division of Bangladesh. It provides inpatient and outpatient medical, surgical, pediatric and gynecology services. Each year there are approximately 700,000 outpatient visits, 700,000 emergency department visits and 600,000 admissions (500,000 adults and 100,000 children). Bed occupancy for medical wards runs at more than $100 \%$ with extra beds provided on a daily basis.
Chittagong is the second largest city of Bangladesh. It is located in southeastern Bangladesh with a population in the metropolitan area of over 6.5 million people. Chittagong has a tropical monsoon climate with average temperatures ranging between 21.6 and $30.2{ }^{\circ} \mathrm{C}$ and average humidity of $78 \%$. The climate varies from tropical monsoon climate from March to November and cool and dry winters from December to February [18].

\section{Patients and clinical methods}

Patients admitted to $\mathrm{CMCH}$ between $15^{\text {th }}$ January 2012 and $5^{\text {th }}$ July 2012 were considered for enrollment. Eligibility criteria were age more than 6 months, documented axillary temperature $\geq 38{ }^{\circ} \mathrm{C}$, a reported history of fever less than 2 weeks, a negative malaria smear and written informed consent given by patient or by the parents or caregiver if a child (age $<16$ years). Each study day all the patients admitted to the "on-take" wards were reviewed and those with a history of fever of the appropriate duration and a negative malaria smear had their temperature measured on at least one occasion. Eligible patients were then asked if they consented to participate in the study. Demographic and clinical information was recorded on a case record form at the time of admission and during the course of hospitalization.

A final diagnosis was made by the study team based on clinical presentation, basic laboratory results and microbiology results. A diagnosis of probable enteric fever was considered if the patient presented with a febrile illness of $>3$ days duration and two or more of the following clinical symptoms: the presence of abdominal symptoms (abdominal pain, diarrhea, constipation, nausea or vomiting), a documented fever of $\geq 39{ }^{\circ} \mathrm{C}$; hepatomegaly and/or splenomegaly; a low or normal white cell count; elevation of liver enzymes (aspartate transaminase, alanine transaminase) three times above the normal range; combined with a slow defervescence with ceftriaxone treatment (the standard antibiotic used for hospital admitted febrile patients with suspected enteric fever) or a clear typhoid complication and no alternative confirmed diagnosis was established.

\section{Laboratory methods}

Blood was taken for complete blood count, urea and creatinine, aspartate transaminase (AST), alanine transaminase (ALT), malaria smear, a single BactAlert ${ }^{\oplus}$ blood culture, and a sample for real time PCR assays and 
serology. Cerebrospinal fluid was taken at the discretion of the responsible physician in suspected central nervous system infection as part of routine practice. Usually only small volumes of CSF were taken, sufficient for a cell count and biochemistry but insufficient for any further investigations. EDTA whole blood, serum, and any residual CSF (where there was sufficient) were stored at $-20{ }^{\circ} \mathrm{C}$ for later analysis by PCR and serology at the Oxford University Clinical Research Unit (OUCRU), Ho Chi Minh City, Vietnam.

\section{Bacterial culture}

Blood was taken for culture within $24 \mathrm{~h}$ of admission, when possible before antimicrobial therapy was started in hospital. A volume of blood, 5-12 mL in adults and 1-12 mL for children, was inoculated into an adult or pediatric BactAlert ${ }^{\bullet}$ blood culture bottle. The exact volume of blood added was determined by weighing the bottle before and after blood inoculation. They were incubated aerobically in a BactAlert ${ }^{\bullet}$ automated system (bioMérieux, Marcy l'Etoile, France) at $37^{\circ} \mathrm{C}$ for 5 days. A Gram stained smear was prepared from the broth of bottles that were positive and which was also sub-cultured onto $5 \%$ sheep blood agar and chocolate agar (Oxoid, Basingstoke, UK) incubated in a candle jar and MacConkey agar (Oxoid, Basingstoke, UK) incubated in air for $48 \mathrm{~h}$. Bacterial isolates were identified by standard methods including biochemical test using API test strips (bioMérieux, Marcy l'Etoile, France) and agglutination with specific antisera (Biorad, Hemel Hempstead, UK) [19].

Antimicrobial susceptibility tests were determined using disc diffusion with results interpreted according to Clinical Laboratory Standards Institute (CLSI) guidelines [20]. Isolates of Salmonella were tested with chloramphenicol $(30 \mu \mathrm{g})$, ampicillin $(10 \mu \mathrm{g})$, trimethoprimsulphamethoxazole $(1.25 / 23.75 \mu \mathrm{g})$, ceftriaxone $(30 \mu \mathrm{g})$, ciprofloxacin $(5 \mu \mathrm{g})$, and nalidixic acid $(30 \mu \mathrm{g})$. The minimum inhibitory concentration (MIC) was determined by E- test according to the manufacturer's guidelines (AB Biodisk, Solna, Sweden) against ciprofloxacin, ceftriaxone and azithromycin. Salmonella breakpoints for ciprofloxacin were: susceptible $\leq 0.06 \mu \mathrm{g} / \mathrm{mL}$; intermediate $>0.06-\leq 0.5 \mu \mathrm{g} / \mathrm{mL}$; and resistant $\geq 1.0 \mu \mathrm{g} / \mathrm{mL}$. A cut-off for susceptibility of $\leq 16 \mu \mathrm{g} / \mathrm{mL}$ was used for azithromycin. Other Enterobacteriacae were additionally tested against ceftazidime $(30 \mu \mathrm{g})$, imipenem $(10 \mu \mathrm{g})$ and gentamicin $(10 \mu \mathrm{g})$. Isolates non-susceptible to ceftriaxone or ceftazidime were tested for extended spectrum beta-lactamase (ESBL) activity by comparing inhibition zone sizes of cefpodoxime, ceftriaxone and ceftazidime with and without clavulanic acid with a difference of $5 \mathrm{~mm}$ or more indicating ESBL activity. Streptococcus pneumoniae was tested with oxacillin $(1 \mu \mathrm{g})$. and Staphylococcus aureus was tested with penicillin (10U) and cefoxitin $(30 \mu \mathrm{g})$ (resistance indicating meticillin resistance). Escherichia coli ATCC25922 and Staphylococcus aureus ATCC25923 were used as control strains for these assays. All media and tests were subject to regular internal quality assessment. Bacterial isolates were stored on beads in glycerol at $-80{ }^{\circ} \mathrm{C}$ and later transferred to the OUCRU, Vietnam for re-confirmation of their identification and susceptibility results.

\section{Serology}

The admission serum was analysed by ELISA for dengue NS1 antigen using a PanBio Kit (PanBio, Sinnamon Park, Australia) according to the manufacturer's instructions.

\section{Nucleic acid amplification tests}

DNA was extracted from the stored whole blood samples $(2 \mathrm{~mL}$ in adults and $1 \mathrm{~mL}$ from children) with a QIAmp DNA mini kit (Qiagen, Manchester, UK). A real-time PCR for S.enterica Typhi and S.enterica Paratyphi was performed using $25 \mu \mathrm{L}$ reactions containing $5 \mu \mathrm{L}$ of extracted DNA targeting STY0201 (Putative fimbrial adhesion in S. Typhi CT18) or SSPA2308 (hypothetical protein in SPA AKU-12601) as previously described [21]. Probe-based real-time PCR was also performed on the DNA extracted from blood to detect Leptospira spp, R.typhi and O.tsustugamushi [22-24]. Low-positive plasmid controls determined adequate detection limits of each assay.

Total nucleic acid was isolated from $100 \mu \mathrm{L}$ of CSF specimens using the automated easyMAG ${ }^{\odot}$ system (bioMérieux), and diagnostic PCRs were performed [25]. Four real-time PCR protocols were used for detection of $S$ pneumoniae, $H$ influenzae type B, Neisseria meningitidis, and Streptococcus suis. Real time-PCRs were used to detect herpes simplex virus 1 and 2, varicella zoster virus, enteroviruses (generic and 71-specific) [26], and human parechoviruses (generic).

\section{Analysis}

This was an observational study intended to serve as the basis for future studies with larger sample sizes and/or interventions. The true incidence of patients presenting with enteric fever in the $\mathrm{CMCH}$ is unknown. With a sample of at least 250 patients we would be able to estimate a disease prevalence of $5 \%$ with a confidence interval $(\mathrm{CI})$ of $\pm 2.8 \%$.

Demographic and clinical features were described for the whole cohort and within the stratified age categories of $<5$ years, $5-15$ years and $\geq 16$ years. Continuous data were described using median and inter-quartile range and compared using the Mann Whitney $U$-test. Proportions were compared with the Chi-squared test or the Fisher's exact test as appropriate. Age, sex, duration of illness prior to admission to hospital, and the clinical 
syndrome were evaluated for association with in-hospital death through a univariate analysis. A multivariate logistic regression model controlling simultaneously for the effects of confounding included variables associated with the outcome of death $(p<0.10)$ as well as a priori factors age, sex and duration of illness prior to admission. Analysis was performed using SPSS version 21 (SPSS inc, Chicago, USA). The dataset supporting the conclusions of this article is available from the corresponding author.

\section{Results}

\section{Demographics and clinical features}

We enrolled 304 eligible febrile patients in this study. It was not possible to take blood from one patient and in three patients, who were blood culture negative, there was insufficient blood for PCR amplification. These four patients were excluded from this analysis. Of the 300 patients analyzed 156 were children (age $\leq 15$ years) and 144 were adults. The median (interquartile range, range) age was $13.5(5.0-31.0,0.5-89)$ years and the median duration of illness before admission was five (IQR, 2-8; range, 1-14) days. A history of prior antimicrobial therapy was reported in 185 (61.7\%) of patients including $96(61.5 \%)$ of children and $89(61.8 \%)$ of adults.

A final clinical suspected or microbiologically confirmed diagnosis of enteric fever was made in 52 (17.3\%; $95 \%$ Confidence Interval 13.5-22.0 \%) patients. The other common clinical syndromes diagnosed were: lower respiratory tract infection in 48 patients $(16.0 \%)$, non-specific febrile illness in 48 patients (16.0\%), a CNS infection in 37 patients (12.3\%), urinary sepsis in 23 patients $(7.7 \%)$, upper respiratory tract infection in 21 patients (7.0\%), and diarrhea (including dysentery) in 21 patients $(7.0 \%)$. Malaria was clinically suspected in seven patients, despite a negative malaria smear, although all had received prior anti-malarial treatment. Two subsequently had a malaria positive rapid diagnostic test result. There were a 14 patients who fulfilled the clinical criteria for enteric fever but had a very rapid response to ceftriaxone treatment that was not typical for enteric fever. They were classified as another syndrome, most commonly as a non-specific febrile illness. The demographic features and clinical syndromes diagnosed in the patients according to age ranges are shown in Table 1.

\section{Microbiological diagnoses}

The median (IQR) volume of blood cultured was 2.2 $(1.7-2.9) \mathrm{mL}$ in children $<5$ years; $5.2(4.2-5.8) \mathrm{mL}$ in children 5-9 years; 6.6 (5.5-9.9) in children $10-15$ years; and $9.2(8.4-10.3)$ in those $\geq 16$ years. A microbiological diagnosis was confirmed in 57 (19\%) patients as outlined in Table 2. All of the positive blood cultures grew just one organism. Salmonella Typhi was most prevalent organism detected in this study and accounted for 34 cases (11.3\%; 95\%CI 8.2-15.5\%). Nineteen (6.3\%) patients had a positive blood culture for $S$. Typhi, including three patients also PCR amplification positive for S.Typhi in blood. Fifteen (5.0 \%) patients with a negative blood culture were PCR amplification positive for $S$. Typhi in blood. The diagnosis was therefore confirmed in $34 / 52(65.4 \%)$ of patients in whom the diagnosis of enteric fever was suspected. No patient was positive for Salmonella Paratyphi A by blood culture or by PCR. A Gram-negative bacillus was found in the blood culture of a patient clinically diagnosed as enteric fever and a Gram positive diplococcus was observed in the blood culture of an adult with pneumonia but neither grew on sub-culture. Two adults were PCR amplification positive for Rickettsia typhi and one was PCR amplification positive for Orientia tsutsugamushi. All patients were PCR amplification negative for Leptospirosis. A further two adults were positive for dengue NS1 antigen in the admission serum sample. In the 37 patients with a clinically diagnosed CNS infection there was sufficient CSF sample available for further examination in 12. These were positive in four children: by PCR for Neiserria meningitidis in two, Streptococcus pneumoniae in one and one was IgM positive for Japanese encephalitis virus.

\section{Antimicrobial susceptibility results}

Antimicrobial susceptibility results were available for 18 of the $S$. Typhi isolates. All 18 had intermediate susceptibility to ciprofloxacin, six were additionally multidrug-resistant (MDR) exhibiting resistance to chloramphenicol, ampicillin and co-trimoxazole but all were susceptible to ceftriaxone and azithromycin. Among the other Enterobacteriacae, one E.coli, one K.pneumoniae and one E.cloacae were resistant to ceftriaxone and Extended Spectrum Beta Lactamase (ESBL) positive. The E.coli and K.pneumoniae were additionally resistant to ciprofloxacin and the K.pneumoniae to gentamicin but all were susceptible to imipenem. The S.pneumoniae was penicillin susceptible and one of the two $S$. aureus was meticillin resistant.

\section{Outcome}

A variety of empirical antimicrobials were employed for treatment. Most patients diagnosed with enteric fever were initially treated with ceftriaxone with a step down to oral azithromycin. The median duration of hospital stay was four (IQR 2-7, range 0-29) days.

A total of $29(9.7 \%)$ patients died during their hospital admission: $15 / 156$ (9.6 \%) of the children and 14/144 $(9.7 \%)$ adults. The mortality was $2 / 52$ (3.8 \%) patients with enteric fever; $5 / 48(10.4 \%)$ patients with lower respiratory tract infection; and $12 / 37$ (32.4 \%) patients 
Table 1 Demographic features and clinical syndromes of 300 patients admitted to CMCH with fever

\begin{tabular}{|c|c|c|c|c|}
\hline Variable $^{a}$ & All & $<5$ years & $5-15$ years & $>15$ years \\
\hline Number & 300 & 70 & 86 & 144 \\
\hline Duration of illness before admission (median (IQR) days) & $5(2-8)$ & $2(1-5)$ & $5(3-10)$ & $5(3-8)$ \\
\hline Male & $173(57.7)$ & $37(52.9)$ & $56(65.1)$ & $80(55.6)$ \\
\hline \multicolumn{5}{|l|}{ Clinical syndrome: } \\
\hline Enteric fever & $52(17.3)$ & $0(0)$ & $19(22.1)$ & $33(22.9)$ \\
\hline Lower respiratory tract infection & $48(16.0)$ & $13(18.6)$ & $5(5.8)$ & $30(20.8)$ \\
\hline Non-specific febrile illness & $48(16.0)$ & $10(14.3)$ & $20(23.3)$ & $18(12.5)$ \\
\hline Central nervous system infection & $37(12.3)$ & $12(17.1)$ & $13(15.1)$ & $12(8.3)$ \\
\hline Urinary tract infection & $23(7.7)$ & $2(2.9)$ & $3(3.5)$ & $18(12.5)$ \\
\hline Upper respiratory tract infection & $21(7.0)$ & $13(18.6)$ & $7(8.1)$ & $1(0.7)$ \\
\hline Diarrhea or dysentery & $21(7.0)$ & $16(22.9)$ & $4(4.7)$ & $1(0.7)$ \\
\hline Malaria & $7(2.3)$ & $0(0)$ & $3(3.5)$ & $4(2.8)$ \\
\hline Hepatobiliary & $7(2.3)$ & $0(0)$ & $4(4.7)$ & $3(2.1)$ \\
\hline Skin and soft tissue infection & $4(1.3)$ & $2(2.9)$ & $0(0)$ & $2(1.4)$ \\
\hline Dengue & $3(1.0)$ & $0(0)$ & $1(1.2)$ & $2(1.4)$ \\
\hline Sepsis syndrome & $3(1.0)$ & $0(0)$ & $0(0)$ & $3(2.1)$ \\
\hline Septic arthritis & $2(0.7)$ & $0(0)$ & $1(1.2)$ & $1(0.7)$ \\
\hline Nephrotic syndrome & $2(0.7)$ & $0(0)$ & $2(2.4)$ & $0(0)$ \\
\hline Dental abscess & $1(0.3)$ & $0(0)$ & $0(0)$ & $1(0.7)$ \\
\hline Other ${ }^{b}$ & $17(5.7)$ & $2(2.9)$ & $4(4.7)$ & $12(8.3)$ \\
\hline Duration of admission (median (IQR) days) & $4(2-8)$ & $4(2-19)$ & $7(4-27)$ & $4(2-5)$ \\
\hline Mortality (Number (\%)) & $29(9.7)$ & $8(11.4)$ & $7(8.1)$ & $14(9.7)$ \\
\hline
\end{tabular}

${ }^{a}$ Results are number (\%) or median (Inter-quartile range)

${ }^{b}$ Haematalogical malignancy (6); autoimmune disease (4); post-streptococcal glomerulonephritis (2); cerebrovascular accident (2); post myocardial infarction (1); suspected brain tumor (1); costochondritis (1)

with encephalitis/meningitis. There was no association with a history of antimicrobial use before hospital admission and mortality $(p>0.5)$. The association of age, sex, duration of illness before admission and the diagnosis of the four commonest clinical syndromes with fatal outcome are shown in Table 3 . In a multivariate analysis including all of these variables a diagnosis of a CNS infection was independently associated with a fatal outcome (OR 6.81 (95\% CI 2.77-16.76; $p<0.001$ ). Of the 12 patients with a CNS infection who died, sufficient CSF was available for pathogen detection in only two of which one was positive for S. pneumoniae.

\section{Discussion}

In this study of febrile adults and children admitted to hospital in Chittagong enteric fever was identified as the most common clinical syndrome responsible for almost one in five admissions. The diagnosis was confirmed by isolation of $S$. Typhi in blood culture and/or by real time PCR amplification from blood for $S$. Typhi in $11 \%$ of admissions and two thirds of suspected cases. Making a confident clinical diagnosis of enteric fever in the absence of laboratory confirmation is difficult. We used a clinical algorithm to define patients with probable enteric fever but excluded a number of patients with a suggestive picture who did not respond to ceftriaxone in the slow manner in which patients with enteric fever often do. We also assumed that the detection of $S$. Typhi DNA in the peripheral blood by the real-time PCR assay indicates active disease although it is possible that the DNA may persist in the blood from a past infection. In the absence of an alternative suitable reference standard it is difficult to be completely confident that these approaches are correct.

Typhoid fever was most commonly diagnosed in school aged children and young adults in this group of hospitalized patients but was not diagnosed in children aged $<5$ years. This is in contrast to community-based studies in other sites in Bangladesh where typhoid was found in children $<5$ years [6-8]. In these communitybased studies with active surveillance by blood cultures of children $<5$ years in clinics, most cases with blood culture confirmed typhoid fever did not require admission to hospital. This may suggest that more severe typhoid disease which requires hospital admission occurs in older age groups. Of the 18 tested S. Typhi 
Table 2 Pathogens detected in 300 adults and children admitted to CMCH with fever

\begin{tabular}{|c|c|c|c|c|}
\hline Pathogens & All & $<5$ years & $5-15$ years & $>15$ years \\
\hline Staphylococcus aureus & 2 & 1 & 1 & 0 \\
\hline Streptococcus pneumoniae & 2 & 1 & 1 & 0 \\
\hline Streptococcus acidominimus & 1 & 0 & 0 & 1 \\
\hline Enterococcus spp. & 1 & 0 & 0 & 1 \\
\hline Escherichia coli & 2 & 0 & 0 & 2 \\
\hline Enterobacter cloacae & 2 & 0 & 0 & 2 \\
\hline Klebsiella pneumoniae & 1 & 0 & 0 & 1 \\
\hline \multicolumn{5}{|c|}{ Salmonella enterica serotype Typhi } \\
\hline Blood culture & 19 & 0 & 8 & 11 \\
\hline PCR & $18^{\mathrm{a}}$ & 0 & 1 & 17 \\
\hline Burkholderia cepacia & 3 & 3 & 0 & 0 \\
\hline Acinetobacter spp. & 1 & 0 & 1 & 0 \\
\hline Neisseria meningitdis & 2 & 0 & 2 & 0 \\
\hline Rickettsia typhi & 2 & 0 & 0 & 2 \\
\hline Orientia tsutsugamushi & 1 & 0 & 0 & 1 \\
\hline Dengue & 2 & 0 & 0 & 2 \\
\hline Japanese encephalitis virus & 1 & 1 & 0 & 0 \\
\hline Total & $57 / 300(19)$ & $6 / 70(9)$ & 13/86 (15) & $38 / 144(26)$ \\
\hline
\end{tabular}

${ }^{\mathrm{a}}$ Three also positive by blood culture

isolates all demonstrated intermediate susceptibility to ciprofloxacin and one third were multidrug resistant. This is consistent with other studies originating in Bangladesh [9-11]. Ceftriaxone and azithromycin remained active and were used for treatment. There were no isolates resistant to ceftriaxone or ciprofloxacin $(\mathrm{MIC} \geq 1.0 \mu \mathrm{g} / \mathrm{mL})[12,13]$. The mortality in the confirmed enteric fever cases at $3.8 \%$ was comparable with other studies of hospitalized patients [27-30]. We did not identify enteric fever caused by $S$. Paratyphi A in this group of patients, however this organism has been isolated from other febrile patients in Chittagong and at other sites in Bangladesh [8]. It may be that paratyphoid fever is less severe than typhoid in this setting and less

Table 3 Univariate analysis of factors associated with a fatal outcome

\begin{tabular}{llll}
\hline Covariate & $\begin{array}{l}\text { Died } \\
n=29\end{array}$ & $\begin{array}{l}\text { Survived } \\
n=271\end{array}$ & $p$ \\
\hline Age (years) $^{\mathrm{a}}$ & $13(4-45)$ & $13(5-30)$ & 0.699 \\
Male $^{\mathrm{b}}$ & $15(51.7)$ & $158(58.3)$ & 0.193 \\
Days ill prior to admission $^{\mathrm{a}}$ & $5(2-8)$ & $4(3-6)$ & 0.713 \\
Enteric fever $^{\mathrm{b}}$ & $2(6.9)$ & $50(18.5)$ & 0.193 \\
Lower respiratory tract infection $^{\mathrm{b}}$ & $5(17.2)$ & $43(15.9)$ & 0.791 \\
Non-specific febrile illness $^{\mathrm{b}}$ & $0(0)$ & $48(17.7)$ & 0.007 \\
Central nervous system infection $^{\mathrm{b}}$ & $12(41.4)$ & $25(9.2)$ & $<0.001$ \\
\hline
\end{tabular}

${ }^{a}$ Median (Inter-quartile range)

${ }^{\mathrm{b}}$ Number (\%) likely to result in hospital admission, although observations across other parts of South Asia suggest that typhoid and paratyphoid may be of equal clinical severity [31].

Lower respiratory tract infections were the second most common clinical syndrome and had a corresponding mortality of $10.4 \%$. A further $15 \%$ of patients presented with non-specific febrile illness and there were no deaths in this group. CNS infections accounted for nearly $12 \%$ of admissions and were responsible for $41 \%$ of deaths. The etiology of these cases was undetermined in most patients mainly because CSF was not available for further analysis. Other identified organisms causing bacteremia included Staphylococcus aureus, Streptococcus pneumoniae, E.coli, Enterobacter cloacae and Klebsiella pneumonia which are typical causes of bacteremia in Asia [32]. The presence of meticillin resistant Staphylococcus aureus (MRSA) and extended spectrum beta-lactamase (ESBL) producing Enterobacteriacae illustrates the importance of resistance surveillance against commonly used antimicrobials in this region [33]. In Bangladesh, antimicrobials can be easily purchased in the community and most patients use them prior to seeking medical attention. More than half of patients in this study consumed one or more antimicrobial prior to a blood draw for microbiological culture. Pre-treatment with antimicrobials will clearly reduce the yield of bacterial pathogens detected in blood and other clinical specimens. The three cases of Burkholderia cepacia bacteremia in children under the 
age of 5 years were unexpected. This organism may be associated with environmental contamination of blood cultures, but in each case here was associated with signs of sepsis.

The detection of two cases of Rickettsia typhi (murine typhus) and one case of Orientia tsustugamushi (scrub typhus) emphasizes the occurrence of these underrecognized pathogens in this area [34]. A case series in Mymensingh in the north of Bangladesh reported 40 Rickettsia infections, this study included 24 patients (60\%) positive for scrub typhus, by the Weil-Felix test [35]. A further case series described seven Bangladeshi nationals in Singapore with murine typhus [36]. In a recent prospective seroepidemiologic survey across six major teaching hospitals in Bangladesh, including $\mathrm{CMCH}$, an IgM enzyme-linked immunosorbent assay was used to detect recent exposure to Rickettsia typhi and Orientia tsustugamushi. The results indicated that 805 of 1209 (66.6 \%) subjects were seropositive for Rickettsia typhi and 287 of 1209 (23.7 \%) were seropositive for Orientia tsutsugamushi [15]. Although the detection of IgM is less specific than IgG and cross-reactivity with antigens of other organisms may potentially lead to an over-estimate of the levels of infection, these studies suggest that these pathogens may be an important and under-reported cause of febrile illness in Bangladesh.

There were no confirmed cases of Burkholderia pseudomallei bacteremia despite previous reports of indigenous cases and in returning travelers $[37,38]$. In a recent seroepidemiologic survey of six hospitals across Bangladesh, 359 of 1244 (28.9 \%) of patients were seropositive for B.pseudomallei by indirect hemagglutination assay suggesting that many people may be exposed to the organism [16]. Also notable by its absence was leptospirosis despite serological evidence that it is a cause of febrile illnesses in this area $[39,40]$. The absence of these infections in this study may be because the surveillance of febrile patients was not conducted throughout the the whole year covering all seasons. This is an important limitation of this study as is the small sample size and the limited range of diagnostic testing performed. The lack of CSF samples for further testing was a particular gap as patients diagnosed with a CNS infection were associated with the highest mortality. The results of this study should be therefore be regarded as preliminary and future studies should incorporate a wider panel of diagnostic methods for relevant pathogens and a minimum of a year-long recruitment period to encompass all potential seasonal variation.

\section{Conclusions}

Enteric fever was a common cause of a non-malaria febrile illness in patients admitted to hospital in Chittagong. All isolated $S$. Typhi exhibited intermediate susceptibility to ciprofloxacin and many were MDR. Infection with $O$. tsutsugamushi and $R$. typhi were additionally confirmed in this setting. Lower respiratory tract and CNS infections were also common and CNS infections had a particularly high mortality rate with more than one third of patients dying during their hospital stay. The etiology of infections in this setting, in particular CNS infections, require further study.

\begin{abstract}
Abbreviations
ALT: Alanine transaminase; API: Analytical profile index; AST: Aspartate transaminase; ATCC: American type culture collection; Cl: Confidence interval; CLSI: Clinical and Laboratory Standards Institute; CMCH: Chittagong Medical College Hospital; CNS: Central nervous system; CSF: Cerebrospinal fluid; DNA: Deoxyribose nucleic acid; EDTA: Ethylenediaminetetraacetic acid; ELISA: Enzyme linked immunosorbent assay; ESBL: Extended-spectrum betalactamase; IgM: Immunoglubulin M; IQR: Inter-quartile range; MDR: Multidrug resistant; MIC: Minimum inhibitory concentration; MRSA: Meticillin resistant Staphylococcus aureus; NS1: Dengue Non-structural protein 1; OUCRU: Oxford University Clinical Research Unit; PCR: Polymerase chain reaction
\end{abstract}

\section{Acknowledgements}

We thank other members of the CMCH Typhoid Study Group: Asok Kumar Dutta, Nasir Uddin Mahmud, Murad Hero, Nafiz labal and Zabeen Chaudhury who recruited patients during the study period; Mr Sanjeeb Kanti Paul and Mr Suman Sharma who performed the malaria microscopy; staff from Chevron Laboratories Ltd. for laboratory support; and Shafiqul Chowdhury for administrative support. We thank the nurses and doctors at Chittagong Medical College Hospital, Chittagong, who were responsible for providing all care for the patients in the study.

\section{Funding}

This project was funded by the Wellcome Trust of Great Britain (106158/Z/ 14/Z) and the European Society for Paediatric Infectious Diseases. Stephen Baker is a Sir Henry Dale Fellow, jointly funded by the Wellcome Trust and the Royal Society (100087/Z/12/Z). Rapeephan R Maude was a Wellcome Trust Masters Training Fellow in Public Health and Tropical Medicine. Hanna $\mathrm{K}$ de Jong was supported by an Academic Medical Center (AMC PhD Scholarship).

\section{Availability of data and materials}

The original data for this study is available from the corresponding author.

\section{Authors' contributions}

RRM, AG, RS, HKdJ, MF, LW, MUH, MAH, MRK, HRVD, RJM, TvdP, WJW, NPD, $S B, A M D, C M P$, MAF conceived and designed the study. RRM, HKdJ, MF, LW, AAS, SVE, SP, ASMZ, WR, RK, RI, TTND, HTT, PHA, JIC, CMP participated in data collection and the analysis and interpretation of data. RRM, HKdJ, SB, CMP, MAF wrote the first draft of the paper. All authors contributed to revising the draft, had full access to all the data and read and approved the final manuscript.

\section{Competing interests}

The authors declare that they have no competing interest.

\section{Consent for publication}

Not applicable.

\section{Ethics approval and consent to participate}

Patients and parents of all children recruited to the study gave witnessed, informed and written consent before study enrolment. The Bangladesh National Research Ethical Committee (BMRC/NREC/2010-2013/1543), Chittagong Medical College Hospital Ethics Committee, the Oxford Tropical Research Ethics Committee (Oxtrec 53-09) and the Research Ethics Committee of the Liverpool School of Tropical Medicine approved the study protocol.

\section{Author details}

'Mahidol-Oxford Tropical Medicine Research Unit (MORU), Faculty of Tropical Medicine, Mahidol University, Bangkok, Thailand. ${ }^{2}$ Chittagong Medical College Hospital, Chittagong, Bangladesh. ${ }^{3}$ Department of Internal Medicine, Division of Infectious Diseases and Center for Infection and Immunity 
Amsterdam (CINIMA), and Center for Experimental Molecular Medicine (CEMM), Academic Medical Center, University of Amsterdam, Amsterdam, The Netherlands. ${ }^{4}$ Clinical Sciences, Liverpool School of Tropical Medicine, Liverpool, UK. ${ }^{5}$ Wellcome Trust Major Overseas Programme, Oxford University Clinical Research Unit (OUCRU), Hospital for Tropical Diseases, Ho Chi Minh City, Vietnam. ${ }^{6}$ Nuffield Department of Medicine, Centre for Tropical Medicine and Global Health, Oxford University, Oxford, UK. ${ }^{7}$ Clinical Research Department, London School of Hygiene and Tropical Medicine, Keppel Street, London WC1E 7HT, UK. ${ }^{8}$ School of Tropical Medicine and Global Health, Nagasaki University, Nagasaki, Japan. ${ }^{9}$ Malaria Research Group and Dev Care Foundation, Chittagong, Bangladesh.

Received: 20 April 2016 Accepted: 1 October 2016 Published online: 13 October 2016

\section{References}

1. Peacock SJ, Newton PN. Public health impact of establishing the cause of bacterial infections in rural Asia. Trans R Soc Trop Med Hyg. 2008;102:5-6.

2. Murdoch DR, Woods CW, Zimmerman MD, Dull PM, Belbase RH, Keenan AJ, et al. The etiology of febrile illness in adults presenting to Patan hospital in Kathmandu, Nepal. Am J Trop Med Hyg. 2004;70:670-5.

3. Phetsouvanh R, Phongmany S, Soukaloun D, Rasachak B, Soukhaseum V, Soukhaseum S, et al. Causes of community-acquired bacteremia and patterns of antimicrobial resistance in Vientiane, Laos. Am J Trop Med Hyg. 2006;75:978-85.

4. Nga TVT, Parry CM, Le T, Lan NPH, Diep TS, Campbell Jl, et al. The decline of typhoid and the rise of non-typhoid salmonellae and fungal infection in a changing HIV landscape: bloodstream infection trends over 15 years in southern Vietnam. Trans R Soc Trop Med Hyg. 2012;106:26-34.

5. Maude R, Hasan MU, Hossain MA, Sayeed AA, Paul SK, Rahman W, et al. Temporal trends in severe malaria in Chittagong, Bangladesh. Malar J. 2012;11: 323.

6. Saha SK, Baqui AH, Hanif M, Darmstadt GL, Rahulamin M, Nagatake T, et al Typhoid fever in Bangladesh: implications for vaccination policy. Pediatr Infect Dis J. 2001;20:521-4.

7. Brookes WA, Hossain A, Goswami D, Nahar K, Alam K, Ahmed N, et al. Bacteremic typhoid fever in children in an urban slum, Bangladesh. Emerg Infect Dis. 2005;11:326-9.

8. Naheed A, Ram PK, Brooks WA, Hossain MA, Parsons MB, Talukder KA, et al. Burden of typhoid and paratyphoid fever in a densely populated urban community, Dhaka, Bangladesh. Int J Infect Dis. 2010;14 Suppl 3:e93-9.

9. Asna SMZH, Haq JA, Rahman MM. Nalidixic acid-resistant Salmonella enterica serovar Typhi with decreased susceptibility to ciprofloxacin caused treatment failure: A report from Bangladesh. Jpn J Infect Dis. 2003;56:32-3.

10. Rahman MM, Haq JA, Morshed MAHG, Rahman MA. Salmonella enterica serovar Typhi with decreased susceptibility to ciprofloxacin. Int J Antimicrob Agents. 2005;25:345-53.

11. Khanam F, Sayeed MA, Choudhury FK, Shiekh A, Ahmed D, Goswami D, et al. Typhoid fever in young children in Bangladesh: Clinical findings, antibiotic susceptibility patterns and immune responses. PLoS Negl Trop Dis. 2015;9:e0003619.

12. Saha SK, Talukder SY, Maksuda I, Saha S. A highly ceftriaxone-resistant Salmonella typhi in Bangladesh. Pediatr Infect Dis J. 1999;18:387.

13. Ahmed D, D'Costa LT, Alam K, Nair GB, Hossain MA. Multidrug-resistant Salmonella enteric serovar Typhi isolates with high-level resistance to ciprofloxacin in Dhaka, Bangladesh. Antimicrob Agents Chemother. 2006;50: 3516-7.

14. Acestor N, Cooksey R, Newton PN, Ménard D, Guerin PJ, Nakagawa J, et al. Mapping the aetiology of non-malarial febrile illness in Southeast Asia through a systematic review - terra incognita impairing treatment policies. PLoS One. 2012;7:e44269.

15. Maude RR, Maude RJ, Ghose A, Amin MR, Islam MB, Ali M, et al. Serosurveillance of Orientia tsutsugamushi and Rickettsia typhi in Bangladesh. Am J Trop Med Hyg. 2014;91:580-3.

16. Maude RR, Maude RJ, Ghose A, Amin MR, Islam MB, Ali M, et al. Seroepidemiological surveillance of Burkholderia pseudomallei in Bangladesh. Trans R Soc Trop Med Hyg. 2012;106:576-8.

17. Maude RR, de Jong HK, Wijedoru L, Fukushima M, Ghose A, Samad R, et al. The diagnostic accuracy of three rapid diagnostic tests for typhoid fever at Chittagong Medical College Hospital, Chittagong, Bangladesh. Trop Med Int Health. 2015;20:1376-84.
18. Peel MC, Finlayson BL, McMahon TA. Updated world map of the KoppenGeiger climate classification. Hydrol Earth Syst Sci. 2007;11:1633-44.

19. Murray PR, Baron EJ, Jorgensen JH, Landry ML, Pfaller MA. Manual of Clinical Microbiology. 9th ed. Washington: American Society of Microbiology; 2007.

20. Clinical and Laboratory Standards Institute. Performance Standards for Antimicrobial Susceptibility Testing: 23rd Informational Supplement. CLSI document M100-S23, vol. 33. Wayne: Clinical and Laboratory Standards Institute; 2013.

21. Nga TV, Karkey A, Dongol S, Thuy HN, Dunstan S, Holt K, et al. The sensitivity of real-time PCR amplification targeting invasive Salmonella serovars in biological specimens. BMC Infect Dis. 2010;10:125.

22. Thaipadunpanit J, Chierakul W, Wuthiekanun V, Limmathurotsakul D, Amornchai $P$, Boonslip $S$, et al. Diagnostic accuracy of real-time PCR assays targeting 16S rRNA and lipl32 genes for human leptospirosis in Thailand: A case-control study. PLoS One. 2011;6:e16236.

23. Jiang J, Chan TC, Temenak JJ, Dasch GA, Ching WM, Richards AL. Development of a quantitative real-time polymerase chain reaction assay specific for Orientia tsutsugamushi. Am J Trop Med Hyg. 2004;70:351-6.

24. Henry KM, Jiang J, Rozmajzl PJ, Azad AF, Macaluso KR, Richards AL. Development of quantitative real-time PCR assays to detect Rickettsia typhi and Rickettsia felis, the causative agents of murine typhus and flea-borne spotted fever. Mol Cell Probes. 2007;21:17-23.

25. Le VT, Phan TQ, Do QH, Nguyen BH, Lam QB, Bach V, et al. Viral etiology of encephalitis in children in southern Vietnam: Results of a one-year prospective descriptive study. Plos Negl Trop Dis. 2010;4:e854.

26. Khanh TH, Sabanathan S, Thanh TT, Le Thoa PK, Thuong TC, Hang VT, et al. A large outbreak of Enterovirus 71 associated hand, foot and mouth disease in southern Vietnam, September-November 2011. Emerg Infect Dis. 2012;18:2002-5.

27. Butler T, Islam A, Kabir I, Jones PK. Patterns of morbidity and mortality in typhoid fever dependent on age and gender: review of 552 hospitalised patients with diarrhea. Rev Infect Dis. 1991;13:85-90.

28. Bhutta ZA. Impact of age and drug resistance on mortality in typhoid fever. Arch Dis Child. 1996;75:214-7.

29. Walia M, Gaind R, Mehta R, Paul P, Aggarwal P, Kalaivani M. Current perspectives of enteric fever: a hospital based study from India. Ann Trop Paediatr. 2005;25:161-74.

30. Parry CM, Thompson C, Vinh H, Chinh NT, Phuong LT, Ho VA, et al. Risk factors for the development of severe typhoid fever in Vietnam. BMC Infect Dis. 2014;14:73.

31. Maskey AP, Day JN, Tuan PQ, Thwaites GE, Campbell Jl, Zimmerman M, et al. Salmonella enterica serovar Paratyphi A and S.enterica serovar Typhi cause indistinguishable clinical syndromes in Kathmandu, Nepal. Clin Infect Dis. 2006:42:1247-53.

32. Deen J, von Seidlein L, Anderson F, Elle N, White NJ, Lubell Y. Community-acquired bacterial blood stream infections in developing countries in south and southeast Asia: a systematic review. Lancet Infect Dis. 2012:12:480-7.

33. Lubell Y, Turner P, Ashley EA, White NJ. Antimicrobial susceptibility of bacterial isolates from community-acquired infections in sub-Saharan African and Asian low and middle income countries. Trop Med Int Health. 2011;16:1967-79.

34. Lobo DA, Velayudhan R, Chatterjee P, Kohli H, Hotez PJ. The neglected tropical diseases of India and South Asia: review of their prevalence, distribution and control or elimination. PLoS Negl Trop Dis. 2011;5:e1222.

35. Miah MT, Rahman S, Sarker CN, Khan GK, Barman TK. Study on 40 cases of Rickettsia. Mymensingh Med J. 2007;16:85-8.

36. Ong AK, Tambyah PA, Ooi S, Kumarasinghe G, Chow C. Endemic typhus in Singapore-a re-emerging infectious disease? Singapore Med J. 2001;42:549-52.

37. Ezzedine K, Malvy D, Steels E, De Dobbeeler G, Struelens M, Jacobs F, et al. Imported melioidosis with an isolated cutaneous presentation in a 90-year-old traveller from Bangladesh. Bull Soc Pathol Exot. 2007;100:22-5.

38. Majumder Ml, Haque MM, Ahmed MW, Alam MN, Rahman MW, Akter F, et al. Melioidosis in an adult male. Mymensingh Med J. 2013;22:413-6.

39. Kendall EA, LaRoccque RC, Bui DM, Galloway R, Ari MD, Goswami D, et al. Leptospirosis as a cause of fever in urban Bangladesh. Am J Trop Med Hyg. 2010;82:1127-30.

40. Swoboda P, Fuehrer HP, Ley B, Starzengruber P, Ley-Threimer K, Jung M, et al. Evidence of a major reservoir of non-malarial febrile diseases in malariaendemic regions of Bangladesh. Am J Trop Med Hyg. 2014;90:377-82 